\title{
Invited Commentary: Applying Psychodynamic Developmental Assessment to Explore Mental Functioning in Adolescents
}

\author{
Shira Tibon Czopp
}

Received: 6 February 2012/ Accepted: 18 February 2012/Published online: 10 March 2012

(C) Springer Science+Business Media, LLC 2012

\begin{abstract}
Recent publications in the Journal of Youth and Adolescence present a variety of topics exploring adolescents' mental functioning in the twenty first century. Conceptually, many of the articles address the intriguing, though rarely explicit, question of developmental continuities and change from adolescence to adulthood. Such investigations, which are particularly prominent in articles that discuss personality dispositions such as impulsivity or lack of empathy and their relationship to emotionally disturbed or maladaptive interpersonal behaviors, examine interactional effects of these dispositions within the personality-context matrix. From a methodological perspective, however, the major tools used for assessing personality dispositions are self-report inventories while performance-based methods, previously defined as projective tests, are not used at all despite the wide range of empirical studies that provide support for their psychometric properties. This commentary suggests that applying a theoretically based, multi-method assessment procedure in empirical research would be most fruitful for any study aimed at exploring mental functioning in adolescents. As an example, I would use the Rorschach Inkblot Method, currently being internationally considered as the most frequently applied personality test for assessing adolescents. Implications for research, practice and policy decisionmaking are discussed.
\end{abstract}

S. Tibon Czopp ( $\square)$

Academic College of Tel-Aviv Yaffo, Tel-Aviv, Israel

e-mail: stibon@mta.ac.il

S. Tibon Czopp

Bar-Ilan University, Ramat-Gan, Israel
Keywords Adolescents - Conduct disorders · Interpersonal functioning - Psychodynamic Diagnostic Manual (PDM) · Rorschach Psychoanalytic Science (RPS)

\section{Introduction}

The Journal of Youth and Adolescence has taken a stance of providing "a single, high-level medium of communication and dialogue for psychologists, psychiatrists, biologists, criminologists, educators, and professionals in many other allied disciplines who address the subject of youth and adolescence". The editorial board stresses the journal's mission as being focused on empirical studies that take into consideration factors relating to developmental sciences and discuss their implications for understanding various aspects of youth experience (Levesque 2011). Recent articles published in the journal present a variety of topics exploring adolescents' mental functioning in the twenty first century. This exploration has made a substantial contribution to issues that are the focus of public concern, current theoretical understanding, empirical research, and clinical and forensic practice with adolescents.

Conceptually, many of the above articles address the intriguing, though rarely explicit, question of developmental continuities and change from adolescence to adulthood. Such investigations examine the effect of personality dispositions such as impulsivity or aggressiveness and their interaction with contextual factors including background characteristics or exposure to trauma, as detecting developmental trends of mental functioning (e.g., Chung et al. 2011; Piquero 2011).

From a methodological perspective, however, the major tools used for assessing both personality dispositions and the different domains of mental functioning are "atheoretical" 
self-report inventories, and to a less extent interviews and/ or theoretical-derived observational measures. Nonetheless, performance-based methods, previously defined as projective tests (e.g., the Rorschach Inkblot Method) are not used at all despite the wide range of empirical studies that consistently provide support for their cross-cultural psychometric properties and incremental validity (Exner 2003; Exner and Erdberg 2005; Meyer 2000, 2001, 2002; Meyer and Viglione 2008; Weiner 2003). This commentary examines the potential utility of using more multi-method assessment tools in the study of adolescence, particularly performance based methods.

\section{Towards a Multi-Method Assessment of Mental Functioning}

Self-report inventories are relatively structured tools that inquire directly about a person's perception of self, state of mind and experiences. In contrast, performance-based methods sample not what people say about themselves but how they perform on various tasks. As an alternative to asking people directly how they perceive themselves, performance-based methods infer these percepts indirectly from their responses to the various tasks. The distinctive features of performance-based methods provide the assessor with clues to personal characteristics and behavioral tendencies of which individuals are not fully aware or are reluctant to disclose. This indirect assessment is less structured and requires an examiner's expertise in administering, coding, and interpreting the test. The codes can nonetheless be quantified to facilitate their interpretation and assessment of their psychometric properties. Being based on both perception of outer reality and projection of inner themes, test responses on performance tasks are interpreted as revealing conscious and unconscious, temporal and longitudinal, structural and dynamic dimensions of mental functioning. The lack of face validity tends to make performance-based methods less susceptible to impression management (Weiner 2008).

With an appreciation for the relative advantages and limitations of both self-report inventories and performancebased methods, assessment psychologists generally conclude that evaluation of mental functioning, which integrates data from different types of tests would paint a more accurate picture of an individual's personality and psychopathology, rather than evaluation consisting only of one of those instruments. This is consistent with recommendations already made by Campbell and Fiske (1959) for multi-method assessment in psychology. Using both types of instruments in clinical, forensic, and educational settings is nonetheless considered as illuminating a wider range of aspects of mental functioning than those that could be revealed by using each of them separately (Bornstein 2010, 2011; Ganellen 2007; Meyer 1997; Weiner 2008).
Despite accumulating clinical experience and empirical evidence that point out the importance of using multi-method assessment in general and with adolescents in particular, this type of research is quite rarely applied. While speculations about the reason for preferring self-report inventories might be related to the nature of the performance-based tests, for example being extremely time consuming, it is also quite likely that the psychoanalytic constructs that these tests are assumed to operationalize have rendered them exiled from the mainstream of academic research. Nonetheless, explorations of psychodynamic performance-based measures substantially have changed their status in the field of assessment. Thus, psychodynamic concepts progressively have given rise to a number of measures derived from performance-based methods. The Ego Impairment Index (EII-2; Viglione et al. 2003) and the Reality-Fantasy Scale (RFS; Tibon et al. 2005), based on the Rorschach Inkblot Method, or the Social Cognition and Object Relations Scale (SCORS; Westen 1991) based on the Thematic Apperception Test (TAT), are some illustrations for these measures. Given these developments, the complaint that empirically validated psychodynamic tools are not used is certainly neither true nor relevant for personality assessment.

\section{Conceptual and Empirical Foundations of Assessing Mental Functioning in Adolescents}

The assessment of mental functioning in adolescents presents a challenging task within the clinical practice. Adolescence constitutes a qualitative new developmental stage of individuation (Bleiberg 2001) in which outside demands frequently induce internal regressive experiences and primitive defensive reactions, sometimes exacerbated by threatening and traumatic events, to which youngsters are particularly sensitive because of the fragility of their personal structure. Since psychiatric disorders commonly emerge during adolescence and some temporarily psychopathological manifestations are more often seen in youngsters, diagnostic issues and treatment planning are particularly important. Additionally, changes in ongoing brain development may result in further emotional turmoil and poor impulse control that might have a substantial effect on maladaptive behaviors (e.g., violent acts, addictions).

In order to classify psychopathological states in adolescents, it is essential to have a working definition of what constitutes age-appropriate healthy functioning at this developmental stage and to collect cross-cultural youngsters' samples of non-patients. These two foundations, the conceptual and the empirical, provide both researchers and clinicians with normative benchmarks for different levels of cognitive and emotional development and are considered to be essential for enabling fruitful research and effective treatment. 
Theoretically, the target of assessing mental functioning is composed of a variety of personality dimensions such as ideation, reality testing, affect modulation, self-perception and level of investment in appropriate and inappropriate interpersonal relationships. A substantial assumption in evaluating youngsters is that, during adolescence, normal developmental demands induce regressive experience and primitive defensive reactions. Accordingly, except for severe psychopathological states, adolescents who show impaired mental functioning on psychological tests might be more reasonably viewed as demonstrating problems evolving from a developmental crisis, sometimes exacerbated by character disturbances and/or trauma-induced reactions, rather than by major mental disorders. These developmental problems, which might include disruptions in cognitive, affective, self-perception and/or interpersonal functioning, can evolve into marked personality disorders in adulthood. Nonetheless, when assessing youth, the clinician should take into consideration that major mental disorders are often present in atypical forms during adolescence. Thus, for example, depression might be masked by eating disorders or severe conduct disorders combined with aggression and reaction to traumatic experience might be demonstrated in psychotic-like manifestations (Exner and Weiner 1995; Tibon and Rothschild 2009; Viglione 1990).

Empirically, psychological tests indicate healthy mental functioning in adolescents when the test results fall within a normative range established in non-patient samples. Results for a test variable that deviate markedly from those of reference samples are likely to indicate maladaptive dysfunction in the aspect of personality functioning measured by this variable. However, this benchmark must be applied while assuming that implications of personality dispositions for psychological adjustment depend on the adolescent's cultural context, consisting of whatever national, ethnic, religious, neighborhood, family, or other group values. Another consideration to be kept in mind is that psychological tests, particularly performance-based methods, do not assess psychopathology directly. Instead, they can be helpful for understanding psychodynamic issues and establishing a differential diagnosis of conditions that are conceptualized in terms of personality dispositions (e.g., impulsivity) that make youngsters prone to develop disturbed interpersonal relationships and the resulting disorders (e.g., Conduct Disorder or Anti-Social Personality Disorder).

\section{Evaluating Proneness to Develop Disturbed Mental Functioning in Adulthood}

An intriguing question is revealed as to developmental continuities from adolescence to adulthood. To address this issue, it is essential to use not only a categorical approach, which offers symptom criteria for diagnoses fitting into the Diagnostic and Statistical Manual of Mental Disorders (DSM; American Psychiatric Association 2000), but also a dimensional diagnostic perspective. The Psychodynamic Diagnostic Manual (PDM Task Force 2006) applies such a dimensional perspective, which is complementary to the $D S M$, assessing the symptoms along a continuum of severity. Recently developed statistical methods enable both practitioners and researchers to distinguish between discrete and continuous phenomena in etiology, assessment and treatment of psychological disorders.

Obviously, the issue of continuities and change from adolescence to adulthood has clinical implications for all types of youth referrals (e.g., somatic symptoms; depressive mood; social phobia). It is nonetheless particularly important in the evaluation of adolescents diagnosed with Conduct Disorder (CD) and those with Post Traumatic Stress Disorder (PTSD), whose potential for developing Anti-Social Personality Disorder (ASPD) and Borderline Personality Disorder (BPD) respectively are in the focus of many studies. Thus, for example, while research exploring ASPD diagnosis generally confirms the assertion that the best predictor of anti-social behavior in adulthood is CD in adolescence (Piquero 2011), it also provides empirical evidence that, although being similar from a phenomenological perspective, CD externalized behavioral manifestations might reflect diverse psychodynamic processes, character problems and psychopathological states. Moreover, although externalized and internalized behaviors are conceptualized as distinct phenomena, many empirical studies including a recent investigation published in this journal (Lee and Stone in press) show that they are closely related and their co-occurrence is mediated by negative self-perception. It is, therefore, essential to assess thoroughly the personality structure of youngsters showing maladaptive externalized behaviors for pursuing diagnostic clarity, especially as to the presence of psychopathic dispositions including deficits in capacity of forming empathic interpersonal relationships that is a core issue in ASPD (e.g., Frick 2004; Gacono and Meloy 1994; McConville and Cornell 2003). Indeed, patterns of social functioning and interpersonal relationships in adolescence usually are considered to have substantial implications when exploring developmental continuities and change in adulthood.

Modern psychoanalytic thinking on patterns of interpersonal relationships refer to these patterns in terms of internalized object relations assuming that the quality of one's interpersonal relations and various types of psychopathology are affected by the maturity of object representations. Assessing object relations should, however, take into account that relational capacity develops progressively and that, in this developmental process, fragmented 
representations gradually turn into complex, differentiated, integrated, and consistent representations of self and objects (Blatt et al. 1976; Leichtman 1996). This process takes place within the context of developmental tasks that reactivate the separation-individuation conflict, searching for the balance between autonomy and relatedness, renegotiating the threat of regressing to dependence and reintegrating new cognitive, social, biological and familial factors. Adolescents who fail to develop mature representations are likely to show any of several interpersonal problems including $\mathrm{CD}$, a mild form of obsessive isolation, manifestations of paranoid style, or a schizotypal disorder (Blatt et al. 1990; Bleiberg 2001).

That a comprehensive conceptualization of mental health is essential for describing disorders and/or maladaptive behaviors may seem self-evident, yet diagnostic procedures in the field have not developed it accordingly. With these challenges in mind, and the frequent discussion of methodological issues shown in prospective studies (Ferguson 2011), this commentary suggests adding psychodynamic-oriented assessment tools, specifically the Rorschach Inkblot Method, to research with adolescents. Capacity for interpersonal relationships is used as an illustration for a major realm of mental functioning that should be assessed thoroughly for evaluating adolescents' proneness to developing psychopathological or maladaptive behaviors in adulthood. It is argued that, similarly to assessment practice in clinical, forensic, and educational settings, applying a multi-method procedure in empirical studies can enable researchers to distinguish between psychopathological conditions including trauma-induced states that might prevail in adulthood and age-based maladaptive behaviors that usually disappear with maturation.

\section{The Utility of the Rorschach Inkblot Method for Psychodynamic Developmental Assessment in Adolescents}

The Rorschach Inkblot Method is considered to be the most frequently used personality test in clinical assessment of adolescents (Archer and Newsom 2000; Perfect et al. 2011). However, as in many fields, practitioners have become increasingly concerned with its reliability and validity. The Comprehensive System (CS; Exner 1974, 2003) provides clinicians with a well-validated and reliable cross-cultural method for scoring and interpreting the Rorschach. Different studies, some of them based on metaanalysis, have confirmed the reliability and validity of Rorschach findings (Society for Personality Assessment 2005), particularly those derived from composite indices (Diener et al. 2011; Meyer 2000; Porcelli and Mihura 2010; Viglione 1999; Viglione and Meyer 2008). Empirical research and analyses of case studies that focus on youngsters provide similar evidence for the utility of the Rorschach in predicting psychopathological manifestations, psychiatric severity and treatment outcomes in children and adolescents (Erdberg 2007; Exner and Weiner 1995; Smith et al. 2001; Tibon and Rothschild 2007; Viglione 1990).

The advance in sophisticated statistical methods necessary to establish the empirical basis for any evidence supported method has gradually diminished the appreciation of conceptual foundations and content analysis of the Rorschach. Thus, the development of the CS with its predominantly statistical emphasis had constituted a real challenge: How can the empirically-based, structurally-derived interpretations be counterbalanced by theoretical concepts and content analysis? Historically, towards the twenty first century, Rorschach work has been changed from being split between empirical and theoretical approaches to more integrative approaches that encompass both empirical evidence and psychodynamic conceptualizations. As to empirical evidence, the Rorschach CS database was widened extensively so as to provide international age-based norms (Meyer et al. 2007). Comparisons between normative data derived from the international project of Meyer et al. (2007) and those collected by Exner (2001) from US adolescents about three decades ago have shown substantial differences with the recent samples looking apparently less adaptive. While the first inclination is to assign psychopathological interpretations, it is quite obvious that such differences might be revealed as a consequence of cultural changes world-wide. Speculation about the cause for the changes over the years might be related to advances in modern technology, including the internet, that must have had an impact on how people interact with the environment. The possible patterns in which that translates to the teen's manner of reacting to the Rorschach is an intriguing area for investigation. The question of whether the differences are due to exposure to environmental changes or to substantial changes in patterns of mental functioning, particularly in adolescence, should be explored further in empirical studies (Tibon Czopp et al. in press).

Based on a psychodynamic developmental paradigm, research pertaining to the various domains of mental functioning, and Rorschach formulations of case studies, this commentary focuses on interpersonal relationships as an illustration for a crucial aspect of assessing adolescents. Psychodynamic developmental conceptualization of interpersonal relationships, as explored by the $P D M$, refers to this realm of mental functioning in terms of object representations. For normative youngsters, object representations become more articulated and more cohesive from mid-adolescence on. The mature inner object world would be revealed in the Rorschach protocol by predominance of 
whole and real human percepts, accurately perceived and fully differentiated from each other, integrating both positive and negative aspects, well articulated, actively motivated, and involved in mutual activities. This type of response points to the capacity of perceiving the object as constant, multidimemsional, and differentiated but nonetheless related to the self (Blatt et al. 1976; Erdberg 2007; Exner and Weiner 1995; Leichtman 1996; Tibon and Rothschild 2007).

Adolescents who fail to develop a mature representational world produce Rorschach protocols with impaired object representations, which tend to deteriorate over time and with stress. The shift from whole to part figures is consistent with the conception that patients with personality disorders might defensively manage the anxiety by splitting the object representations into good and bad ones, failing to integrate apparently contradicted characteristics in the same object (Lerner 1998). The undeveloped inner objects would be revealed in these protocols by an elevated number of responses with vague Developmental Quality (DQv) and predominance of part and/or human-like objects that are less articulated, less accurately perceived and are more deformed, distorted, and destructive. The interaction would usually be malevolent, and lacking mutuality (Blatt et al. 1990; Viglione et al. 2003). Interpersonal relationships would be described as being chaotic and most often saturated by raw, uncontrolled aggressive contents (e.g., Two people cutting each other and you can see the blood on their hands....). The excessive degree of aggression might sometimes reach the point of collapse of potential space (Winnicott 1971) where the blot is experienced as a real object: It's a vicious bat, swooping down to fasten its fang on someone's throat (Smith 1990; Tibon and Rothschild 2007).

Interpersonal functioning is assessed by a variety of Rorschach CS structural variables including the coping deficit index (CDI), the hypervigilance index (HVI), cooperative (COP) and aggressive (AG) responses, and some other variables assumed to measure egocentricity, capacity for empathy, affect modulation, patterns of attachment and human object representations. Some of these measures, such as the Egocentricity Index, were found to distinguish between psychopathic and non-psychopathic adults (Gacono and Meloy 1994). However, adjustments must be made when interpreting these CS measures in adolescents because exceeding the age-based normative range does not necessarily indicate proneness to develop psychopathic tendencies in adulthood (Exner and Weiner 1995).

Apart from these CS variables, different scales and indices that assess the quality of object representations with the Rorschach, including the Concept of Object Relations (COR; Blatt et al. 1976) and the Mutuality of Autonomy Scale (MOA; Urist 1977) have been validated by extensive research on adolescents (Exner and Weiner 1995; Weiner 2008). Applying these Rorschach variables in empirical research with adolescents can broaden our understanding of personality dispositions (e.g., capacity to form cooperative interpersonal relationships) that underline behavioral problems (e.g., aggressiveness), beyond what can be concluded from using self-report measures only. This is certainly true for youngsters seen in clinical, forensic and educational settings as demonstrated in analyses of case studies (Exner and Erdberg 2005), where the question of continuities and change is essential for evaluating treatment outcomes and policy-making decisions.

Variations in Rorschach findings among adolescents with problems in interpersonal functioning have implications for selecting the interventions that would fit into the specific personality-context matrix in which these problems occurred. However, youngsters involved in maladaptive social behaviors are likely to produce different kinds of Rorschach protocols. In some cases, the Rorschach protocol might point out character problems (e.g., limited capacity for impulse control). The extent to which these problems would be translated into anti-social behaviors in adulthood depends on contextual factors. Nonetheless, these behaviors also might be rooted in psychotic or neuropsychological disturbances, and the Rorschach can be extremely useful in detecting the presence of these disturbances.

\section{Conclusion}

This commentary provides an opportunity to assess progress, problems, and prospects of personality assessment research in adolescents. By transporting performancebased tools from clinical practice to empirical inquiry, the commentary is aimed to place psychodynamic-oriented research in adolescents more centrally into the context of developmental science. This is in line with Kandell's (1998) suggestion that experimental psychology in the field of processing and memory provides evidence for psychoanalytic conceptualizations of unconscious phenomena. Accordingly, the essence of the current paradigm might be defined as interpreting findings derived from assessment tools within the context of applied science, relating to psychodynamic processes, normative data, developmental issues, and contextual factors. The purpose of the new framework is to use the unique properties of psychological tests, particularly those previously defined as projective methods, between perception and projection to extend our understanding of healthy versus psychopathological mental functioning in adolescents. It is suggested applying this integrative paradigm in any assessment procedure of adolescents, regardless of the framework in which this procedure is conducted (i.e., research or practice). 
The conceptual foundations and accumulative crosscultural normative data have made the Rorschach Inkblot Method an effective assessment tool for both research and practice in adolescents. Recent empirical findings derived from cognitive and neuropsychological studies (e.g., Spence et al. 2009; Vecera et al. 2002) have enabled researchers to relate the unique properties of the Rorschach task to these findings, and enhance our understanding about the brain-mind matrix. Thus, for example, Giromini et al. (2010) have shown that the mirror neuron system (MNS) can be activated by Rorschach cards, representing static, ambiguous stimuli. This suggests that projective material, such as internal representation of the "feeling of movement," may be sufficient to trigger MNS activity even when minimal external cues are present.

Applying an integrative approach to Rorschach work demonstrates the manner in which psychoanalytic theory and empirical research can be used to conceptualize and synthesize psychological assessment data (Bornstein 2010, 2011) and to use them for understanding psychopathological or maladaptive behaviors as being rooted in personality structure (Gabbard 2006). Such an approach fits into the model of Rorschach Psychoanalytic Science (RPS; Tibon Czopp 2011), which is particularly recommended to be used for evaluating adolescents' mental functioning.

The commentary illustrates the application of the RPS model for assessing interpersonal relationships as a major factor predicting behavioral problems in adulthood. When the behavioral picture of an adolescent points out difficulties in interpersonal relationships, distinguishing between problems derived from emotional disturbance and those reflecting social maladjustment and not psychopathological phenomena might have significant implications for treatment and policy-making decisions. Thus, for example, in some US school districts, findings of emotional disturbance as opposed to social maladjustment are most important for decisions about the availability of special education services (Exner and Erdberg 2005). However, in many countries there are formal guidelines that delineate specific criteria for emotional disturbance and require that it would be differentiated from social maladjusted behavior. Although there has been significant controversy as to whether psychopathology and maladaptive behavior are two distinct categories, this requirement excludes children from special education services if their difficulties solely involve social maladjustment. Making this differentiation is a challenging task for clinicians who are expected to provide psychodynamic personality assessment of adolescents.

Indeed, personality assessment as a field of study is in a particularly tricky position with its threatened identity as a science, sometimes accepted as such but often not. We know that within a context where publication in high impact-factor journals has such important consequences, there is very strong pressure to demonstrate scientific credentials. Certainly, the notion of evidence-based personality assessment should be firmly supported. However, it is suspected that the way most researchers seek to do this undermines the need and the ability to conduct systematic conceptualized investigations of our specific object of study - the human subject. Specifically, the danger is when researchers focus on selecting methods that can easily be defined as being scientifically supported rather than searching for those providing conceptual implications, thus enabling researchers to explore the relationship between what is studied and how it is studied. This might be revealed in some type of rigidity in research, which limits rather than facilitates the progress of the field.

It should be stressed that applying performance-based methods does not exclude self-report inventories as part of the assessment tools. Thus, it is not argued that self-report measures per se should be excluded from research in personality assessment (if it were, this would imply arguing against many studies accepted for publication in this journal). Instead, it is argued that while these measures are most useful for assessing conscious personality characteristics, exclusive reliance on them constrains the way that we can conceptualize the human subject and, as a result, limits the empirical investigation of more dynamic perspectives. The use of self-report measures as the overly dominant tool runs the risk that rather than method being the practice of theory, theory becomes a reflection of method. In line with editorial suggestions as to future submissions to this journal (Levesque 2007), this commentary points to the potential benefits of using evidencebased psychodynamic-oriented tools, specifically the Rorschach Inkblot Method, for a coherent, integrative research in adolescents. The unique properties of the Rorschach are particularly useful for extending our understanding of adolescents' mental functioning and providing an effective tool for distinguishing between emotional disturbance and maladjustment that might be revealed in similar behavioral problems. The differentiation between psychopathology and maladjustment as the source of behavioral difficulties is considered to be crucial for the question of continuities and change from adolescence to adulthood.

We might conclude with some thoughts of what can be done within this field of research in order to conserve the tradition of serving as a force for understanding adolescents. There is a danger that we apply what we consider to be the norms (i.e. using self-rating measures). Accordingly, in published research we are tempted to simply keep providing a forum for a diversity of studies that use the acceptable type of tools and are coherent as to the questions they pose and the procedures they use for answering them. However, it might be fruitful to take the risk of adding empirical research with less popular and more time 
consuming tools that explore mental functioning in adolescents by applying psychodynamic developmental assessment, even though this perspective on mental functioning essentially has been exiled from what is called psychological academic thinking. Research topics on the period of adolescence are migrating outside of academia and the boundaries of our field of research are blurring. If some of the published articles follow these trends, a start would be made for bridging the gap between academia and practice.

While recognizing that just as psychodynamic developmental assessment seems to have an uncertain location among both clinicians and researchers, currently there seems to be much more openness and enthusiasm to what empirically supported performance-based assessment tools can contribute to the research in adolescents. It is hard to foresee how these changes would play out, but they need to be debated now, and the peer-review journals in the field can provide an excellent location to start with. Should these journals focus more tightly and narrowly and just include work that might be familiar to and easily celebrated by psychological science? Some would disagree, but I think that applying a broader range of methods for assessing mental functioning would make the published research more applicable for practitioners engaged in understanding adolescence as a distinctive developmental stage with all its complexities. Could these journals become a site that aims to grasp the new challenge of exploring and communicating in genuinely interdisciplinary field? The answer depends on future submissions as much as it does on the responses of colleagues who would use this exploration.

Acknowledgments I would like to thank Roger J. R. Levesque, Editor-in-Chief, Journal of Youth and Adolescence, who invited me to submit this comment, for his useful suggestions concerning previous versions of this manuscript.

\section{References}

American Psychiatric Association. (2000). Diagnostic and statistical manual of mental disorders (DSM-IV-TR). Washington, DC: Author.

Archer, R. P., \& Newsom, C. R. (2000). Psychological test usage with adolescent clients: Survey update. Assessment, 7, 227-235.

Blatt, S. J., Brenneis, C. B., Schimek, J. G., \& Glick, M. (1976). Normal development and psychopathological impairment of the concept of the object on the Rorschach. Journal of Abnormal Psychology, 85, 364-373.

Blatt, S. J., Tuber, S., \& Auerbach, J. (1990). Representation of interpersonal interaction on the Rorschach and level of psychopathological impairment. Journal of Personality Assessment, 54, $711-728$

Bleiberg, E. (2001). Treating personality disorders in children and adolescents: A relational approach. New York: Guilford Press.

Bornstein, R. F. (2010). Psychoanalytic theory as a unifying framework for 21 st century personality assessment. Psychoanalytic Psychology, 27, 133-152.
Bornstein, R. F. (2011). From symptom to process: How the PDM alters goals and strategies in psychological assessment. Journal of Personality Assessment, 93, 142-150.

Campbell, D. T., \& Fiske, D. W. (1959). Convergent and discriminant validation by the multitrait-multimethod matrix. Psychological Bulletin, 56, 81-105.

Chung, H., Mulvey, E., \& Steinberg, L. (2011). Understanding the school outcomes of juvenile offenders: An exploration of neighborhood influences and motivational resources. Journal of Youth and Adolescence, 40, 1025-1038.

Diener, M. J., Hilsenroth, M. J., Shaffer, S. A., \& Sexton, J. E. (2011). A metaanalysis of the relationships between the Rorschach Ego Impairment Index (EII) and Psychiatric Severity. Clinical Psychology and Psychotherapy, 18, 464-485.

Erdberg, P. (2007). Using the Rorschach in children. In S. R. Smith \& L. Handler (Eds.), The clinical assessment of children and adolescents: A practitioner's handbook. NJ: Lawrence Erlbaum.

Exner, J. E. (1974). The Rorschach: A comprehensive system, Vol. 1. Basic foundation (Vol. 1). NY: Wiley.

Exner, J. E. (2001). A Rorschach workbook for the comprehensive system (5th ed.). Asheville, NC: Rorschach Workshops.

Exner, J. E. (2003). The Rorschach: A comprehensive system, Vol. 1. Basic Foundations (4th ed.). NY: Wiley.

Exner, J. E., \& Erdberg, P. (2005). The Rorschach: A comprehensive system: Vol. 2: Advanced interpretation (3rd ed.). Hoboken, NJ: Wiley.

Exner, J. E., \& Weiner, I. B. (1995). The Rorschach: A comprehensive system, Vol. 3. Assessment of children and adolescents (2nd ed.). NY: Wiley.

Ferguson, C. J. (2011). Video games and youth violence: A prospective analysis in adolescents. Journal of Youth and Adolescence, 40, 377-391.

Frick, P. (2004). Developmental pathways to conduct disorder: Implications for serving youth who show severe aggressive and antisocial behavior. Psychology in Schools, 41, 819-834.

Gabbard, G. O. (2006). Psychodynamic psychiatry in clinical practice (4th ed.). Washington, DC: American Psychiatric Publishing.

Gacono, C. B., \& Meloy, J. R. (1994). The Rorschach assessment of aggressive and psychopathic personalities. Hillsdale, NJ: Lawrence Erlbaum Associates.

Ganellen, R. J. (2007). Assessing normal and abnormal personality functioning: Strengths and weaknesses of self-report, observer, and performance-based methods. Journal of Personality Assessment, 89, 30-40.

Giromini, L., Porcelli, P., Parolin, L., Viglione, J. D., \& Pinedea, J. A. (2010). The feeling of movement: EEG evidence for mirroring activity during the observations of static, ambiguous, stimulus in the Rorschach cards. Biological Psychology, 85, 233-241.

Kandell, E. R. (1998). A new intellectual framework for psychiatry. American Journal of Psychiatry, 155, 457-469.

Lee, E., \& Stone, S. I. (in press). Co-occurring internalized and externalized behavioral problems: The mediating effect of negative self-concept. Journal of Youth and Adolescence.

Leichtman, M. B. (1996). The Rorschach: A developmental perspective. Hillsdale, NJ: The Analytic Press.

Lerner, P. M. (1998). Psychoanalytic perspectives on the Rorschach. Hillsdale, NJ: The Analytic Press.

Levesque, J. R. (2007). Reporting criminal research on adolescence: Reflections on the appeal of coherence, purpose, and responsibility. Journal of Youth and Adolescence, 36, 1-9.

Levesque, J. R. (2011). The journal of youth and adolescence at 40. Journal of Youth and Adolescence, 40, 1-2.

McConville, D., \& Cornell, D. (2003). Aggressive attitudes predict aggressive behavior in middle school students. Journal of Emotional and Behavioral Disorders, 11, 179-187. 
Meyer, G. J. (1997). On the integration of personality assessment methods: The Rorschach and MMPI. Journal of Personality Assessment, 68, 297-330.

Meyer, G. J. (2000). The incremental validity of the Rorschach Prognostic Rating Scale over the MMPI Ego Strength Scale and IQ. Journal of Personality Assessment, 74, 356-370.

Meyer, J. G. (2001). Evidence to correct misperceptions about Rorschach norms. Clinical Psychology: Science and Practice, 8, 389-396.

Meyer, G. J. (2002). Exploring possible ethnic differences and bias in the Rorschach comprehensive system. Journal of Personality Assessment, 78, 104-129.

Meyer, G. J., Erdberg, P., \& Shaffer, T. W. (2007). Toward international normative reference data for the comprehensive system. Journal of Personality Assessment, 89, S201-S216.

Meyer, G. J., \& Viglione, D. J. (2008). An introduction to Rorschach assessment. In R. P. Archer \& S. R. Smith (Eds.), Personality assessment (pp. 281-336). New York, NY: Routledge.

PDM Task Force. (2006). Psychodynamic diagnostic manual. Silver Spring, MD: Alliance of Psychoanalytic Organizations.

Perfect, M. M., Tharinger, D. J., Keth, T. Z., \& Lyle-Lahroud, T. (2011). Relations between MMPI-A scales and severity of maltreatment among adolescents. Journal of Personality Assessment, 93, 582-591.

Piquero, A. R. (2011). James Joyce, Alice in Wonderland, the Rolling Stones, and Criminal Careers. Journal of Youth and Adolescence, 40, 761-775.

Porcelli, P., \& Mihura, J. L. (2010). Assessment of alexithymia with the Rorschach comprehensive system: The Rorschach Alexithymia Scale (RAS). Journal of Personality Assessment, 92, $128-136$

Smith, B. (1990). Potential space and the Rorschach: Application of object relations theory. Journal of Personality Assessment, 55, $756-767$.

Smith, S. P., Baity, M. R., Knowles, E. S., \& Hilsenroth, M. J. (2001). Assessment of disordered thinking in children and adolescents: The Rorschach perceptual thinking index. Journal of Personality Assessment, 77, 447-463.

Society for Personality Assessment. (2005). The status of the Rorschach in clinical and forensic practice: An official statement by the board of trustees of the society for personality assessment. Journal of Personality Assessment, 85, 219-237.

Spence, S. A., et al. (2009). Toward a cognitive neurobiological account of free association. Neuropsychoanalysis, 11, 151-153.

Tibon Czopp, S. (2011). Rorschach inkblot method. In J. R. Levesque (Ed.), Encyclopedia of adolescence (Vol. 4, pp. 2411-2421). New York, NY: Springer.

Tibon Czopp, S., Rothschild Yakar, L., \& Appel, L. (in press). Rorschach comprehensive system (CS) reference data for Israeli adolescents. Journal for Personality Assessment.

Tibon, S., Handelzalts, J. E., \& Weinberger, Y. (2005). Using the Rorschach for exploring the concept of transitional space within the political context of the Middle East. International Journal of Applied Psychoanalytic Studies, 2, 40-57.

Tibon, S., \& Rothschild, R. (2007). Rorschach case formulation in adolescents: A psychoanalytic perspective on the comprehensive system. In S. R. Smith \& L. Handler (Eds.), The clinical assessment of children and adolescents: A practitioner's handbook. NJ: Lawrence Erlbaum.

Tibon, S., \& Rothschild, L. (2009). Dissociative states in eating disorders: An empirical Rorschach study. Psychoanalytic Psychology, 26, 69-82.

Urist, J. (1977). The Rorschach test and the assessment of object relations. Journal of Personality Assessment, 41, 3-9.

Vecera, S. P., Vogel, E. K., \& Woodman, G. F. (2002). Lower-region: A new cue for figure-ground assignment. Journal of Experimental Psychology: General, 131, 194-205.

Viglione, D. J. (1990). Severe disturbance or trauma-induced adaptive reaction: A Rorschach child case study. Journal of Personality Assessment, 55, 280-295.

Viglione, D. J. (1999). A review of recent research addressing the utility of the Rorschach. Psychological Assessment, 11, 251-265.

Viglione, D. J., \& Meyer, G. J. (2008). An overview of Rorschach psychometrics for forensic practice. In C. B. Gacono \& F. B. Evans (Eds.), Handbook of forensic Rorschach assessment (pp. 21-54). New York: Routledge.

Viglione, D. J., Perry, W., \& Meyer, G. J. (2003). Refinements in the Rorschach ego impairment index incorporating the human representational variable. Journal of Personality Assessment, 8, $149-156$.

Weiner, I. B. (2003). Principles of Rorschach interpretation. Mahwah, NJ: Lawrence Erlbaum Associates, Inc.

Weiner, I. B. (2008). The Rorschach inkblot method. In I. B. Weiner \& R. L. Greene (Eds.), Handbook of personality assessment. Hoboken, NJ: Wiley.

Westen, D. (1991). Social cognition and object relations. Psychological Bulletin, 109, 429-455.

Winnicott, D. W. (1971). Playing and reality. New York: Basic books.

\section{Author Biography}

Shira Tibon Czopp is an Associate Professor at the Academic College of Tel-Aviv Yaffo and Bar-Ilan University, Israel. She received her Ph.D. in 1998 from the University of London UK, and the diploma of the American Board of Assessment Psychology and the American Academy of Assessment Psychology in 2001. Her major research interests include psychodynamic assessment and psychotherapy, exploring cross-cultural normative data of the Rorschach Inkblot Method, evaluating the effects of exposure to terror threat on mental functioning, and assessing mental disorders in adolescence. 